\title{
ON THE COEFFICIENTS AND THE GROWTH OF GAP POWER SERIES*
}

\author{
DIETER GAIER $\dagger$
}

\section{Introduction and outline of general method.}

1.1. Introduction. Assume that we are given an entire function $f$ with a gap power series expansion, i.e.,

$$
\text { (1.1) } f(z)=\sum_{n=0}^{\infty} a_{n} z^{n} \text { with } a_{n}=0 \text { for } n \neq \lambda_{k}, k=1,2, \cdots \text {, }
$$

where $\left\{\lambda_{k}\right\}$ is a certain sequence of natural numbers, $0<\lambda_{1}<\lambda_{2}<\cdots$. It is well-known that under suitable conditions on $\left\{\lambda_{k}\right\}$ the function $f$ has, roughly speaking, about the same rate of growth as $z \rightarrow \infty$ in different directions. For example [11, p. 622], if $\left\{\lambda_{k}\right\}$ has density $D \geqq 0$, then in every angle $\alpha \leqq \arg z \leqq \beta$ with $\beta-\alpha>2 \pi D, f$ will be of the same order and type as in the full plane. In particular, if the power series has Fabry gaps:

$$
D=0, \quad \text { or equivalently } \quad \frac{\lambda_{k}}{k} \rightarrow \infty, \quad k \rightarrow \infty,
$$

we can conclude from order and type in every angle $\alpha \leqq \arg z \leqq \beta, \beta>\alpha$, to the order and type of $f$ in the full plane.

In this paper we are interested in the limiting case, in which not the behavior of $f$ in an angle, but only on a radius, for example for $z=x>0$, is known. Fabry gaps no longer suffice to get information about the growth of $m(r)=\max _{|z|=r}|f(z)|$, since already Pólya pointed out [11, p. 636] that there exist entire functions with Fabry gaps (even $\lambda_{k} / k \geqq \log \log k$ ), which are bounded for $x>0$.

Instead, it will be seen that the slightly stronger gap condition

$$
a_{n}=0 \text { for } n \neq \lambda_{k}, \quad k=1,2, \cdots \text {, with } \sum_{k=1}^{\infty} \frac{1}{\lambda_{k}}<\infty,
$$

will be the proper one to conclude from the growth of $f$ on $z=x>0$ to a similar growth of $m(r)$. One particular case is known:

Theorem [9, p. 286]. If the entire function (1.1) satisfies the gap condidition (1.2), and $f(z)$ is bounded for $z=x>0$, then $f$ is a constant; in fact, $f=0$ since $a_{0}=0$.

* Received by the editors April 2, 1965.

Dedicated to Professor J. L. Walsh on the occasion of his seventieth birthday.

$\dagger$ Mathematisches Institut der Universität, Giessen, Germany, and California Institute of Technology, Pasadena, California. This research was supported in part by the Office of Naval Research. 
One of our results will be:

Theorem 6 . If the entire function (1.1) satisfies the gap condition (1.2) and if $f(x)=O\left(e^{x^{\alpha}}\right), x \rightarrow+\infty, \alpha>0$, then $f$ is at most of order $\alpha$ and type 1 .

A theorem of this type was recently proved by the author [5]. There the growth of $f$ on an arbitrary Jordan arc from 0 to $\infty$ (instead of $z=x>0$ ) was prescribed, but the gap condition (1.2) had to be strengthened to

$$
\frac{\lambda_{k}}{k(\log k)^{2+\epsilon}} \rightarrow \infty \quad k \rightarrow \infty, \text { some } \epsilon>0,
$$

in order to apply the Wiman-Valiron theory. The case $\alpha=1$ plays a decisive role in the proof of the unrestricted high indices theorem for Borel summability and motivated our investigations.

A main step in our proof will be the derivation of a representation formula for the coefficients $a_{n}$ of $f$ (see (1.11)), and the radial growth of $f$ will be reflected in an estimate of $\left|a_{n}\right|$, which in turn can be used to estimate $m(r)$. Such estimates of $\left|a_{n}\right|$ are typical for high indices theorems for power series, but our complex variable method does not give such fine estimates as $a_{n}=O(1)$ for a Hadamard gap power series in $|z|<1$ which is bounded on $(0,1)$. We quote one of our results in this direction.

Theorem 11. If $f(z)=\sum_{n=0}^{\infty} a_{n} z^{n}$ is regular in $|z|<1$ and has Hadamard gaps, i.e.,

$$
a_{n}=0 \text { for } n \neq \lambda_{k}, \text { where } \frac{\lambda_{k+1}}{\lambda_{k}} \geqq \theta>1,
$$

then each of the conditions

$f(x)=s+O\left((1-x)^{\alpha}\right), x \rightarrow 1-0, \alpha>0$, or $f^{\prime} \in L_{p}(0,1), p>1$, implies $\sum_{n=0}^{\infty}\left|a_{n}\right|^{\epsilon}<\infty$ for every $\epsilon>0$.

1.2. Lemma on functions of exponential type. The following lemma of Phragmén-Lindelöf type will be used.

Lemma 1 [12, p. 36], [2, p. 82]. Let $f$ be regular and of exponential type in $\operatorname{Re} z \geqq 0,|f(z)| \leqq M$ for $z=i y$, and

Then

$$
h_{f}(0)=\limsup _{x \rightarrow+\infty} \frac{\log |f(x)|}{x} \leqq c .
$$

$$
|f(z)| \leqq M e^{c x}, \quad z=x+i y, x \geqq 0 .
$$

1.3. Outline of general method. Assume that $f(z)=\sum_{n=0}^{\infty} a_{n} z^{n}$ converges for $|z|<T_{0}, 0<T_{0} \leqq \infty$, and that $a_{0}=0$. For any fixed $T$ with $0<T<T_{0}$ we shall study the auxiliary function

$$
H(z ; T)=\int_{0}^{T} f(t) t^{-z-1} d t, \quad z=x+i y .
$$


This transformation (with $T=\infty$ ) has been employed by Edrei [4, p. 121] in the case that $f(t)$ was bounded on $t>0$, but the corresponding transformation for Dirichlet series (see (4.3)) goes back to V. Bernstein [1, p. 111] who used it for different purposes. Since $f(t) / t$ is regular on $\langle 0, T\rangle$, $H(z ; T)$ will be defined for $\operatorname{Re} z<1$, and will represent there a regular function.

On the imaginary axis we have

$$
|H(z ; T)| \leqq \int_{0}^{T} \frac{|f(t)|}{t} d t=M(T), \quad z=i y .
$$

In order to obtain the analytic continuation of $H(z ; T)$ beyond $\operatorname{Re} z=1$, we write for $z$ in $\operatorname{Re} z<0$ (so that $\left|t^{-z}\right|$ is bounded on $\langle 0, T\rangle$ )

$$
H(z ; T)=\int_{0}^{T} \sum_{1}^{\infty} a_{n} t^{n-z-1} d t=\sum_{1}^{\infty} a_{n} \int_{0}^{T} t^{n-z-1} d t=\sum_{1}^{\infty} a_{n} \frac{T^{n-z}}{n-z},
$$

so that $H(z ; T)$ has the alternate representation in $\operatorname{Re} z<0$ :

$$
H(z ; T)=-T^{-z} \cdot \sum_{n=1}^{\infty} \frac{a_{n} T^{n}}{z-n} .
$$

However, since $D(T)=\sum_{n=1}^{\infty}\left|a_{n}\right| T^{n}<\infty$, the series in (1.6) converges uniformly for all $z$ with $|z-n| \geqq \eta>0$, i.e., $H(z ; T)$ is a meromorphic function with possible simple poles at $z=n$, at which $H(z ; T)$ has residues $-a_{n}, n=1,2, \cdots$.

As for the growth of $H$, we immediately obtain from (1.6)

$$
\begin{aligned}
|H(z ; T)| \leqq T^{-x} \cdot \frac{D(T)}{\eta} \text { if } \quad|z-n| \geqq \eta & >0 \\
& \text { for } n=1,2, \cdots .
\end{aligned}
$$

From now on we shall assume that

$$
a_{n}=0 \text { for } n \neq \lambda_{k} \text {, with } \sum_{k=1}^{\infty} \frac{1}{\lambda_{k}}<\infty .
$$

We form the Blaschke product

$$
B(z)=\prod_{k=1}^{\infty} \frac{\lambda_{k}-z}{\lambda_{k}+z}=\prod_{k=1}^{\infty}\left(1-\frac{2 z}{\lambda_{k}+z}\right),
$$

which converges for every $z \neq-\lambda_{k}, k=1,2, \cdots . B(z)$ has simple zeros at $z=\lambda_{k}$, simple poles at $z=-\lambda_{k}$, and

(1.9) $|B(z)| \leqq 1$ for $\operatorname{Re} z \geqq 0$ and $|B(z)|=1$ for $\operatorname{Re} z=0$.

Therefore

$$
\Phi(z ; T)=B(z) \cdot H(z ; T)
$$


is regular in $\operatorname{Re} z \geqq 0$, and $|\Phi(z ; T)| \leqq M(T)$ for $z=i y$. With regards to the growth of $\Phi$ in $\operatorname{Re} z \geqq 0$, we get from (1.7)

$$
|\Phi(z ; T)| \leqq 2 D(T) \cdot T^{-x} \text { if }|z-n| \geqq \frac{1}{2}, \quad n=1,2, \cdots ;
$$

thus on the circle $|z-n|=1 / 2$ we have

$$
|\Phi(z ; T)| \leqq 2 D(T) T^{-n} \cdot\left\{\begin{array}{l}
T^{1 / 2} \text { if } T \geqq 1, \\
T^{-1 / 2} \text { if } T<1,
\end{array}\right.
$$

which by the maximum principle holds also inside that circle. This implies

$$
|\Phi(z ; T)| \leqq D^{\prime}(T) \cdot T^{-x}, \quad \operatorname{Re} z \geqq 0,
$$

with $D^{\prime}(T)=2 D(T) \cdot T^{ \pm 1}$, depending on whether $T \geqq 1$ or $T<1$.

This estimate of $|\Phi|$, which contains the "bad" constant $D(T)$ $=\sum\left|a_{n}\right| T^{n}$, can be improved by an application of Lemma 1. First, (1.10) shows that $\Phi$ is of exponential type in $\operatorname{Re} z \geqq 0$, and furthermore

$$
h_{\Phi}(0)=\limsup _{x \rightarrow+\infty} \frac{\log |\Phi(x ; T)|}{x} \leqq-\log T .
$$

Lemma 1 yields therefore $|\Phi(z ; T)| \leqq M(T) e^{\left(-\log T^{T}\right) x}, \operatorname{Re} z=x \geqq 0$, and for $z=n=\lambda_{m}$ we obtain, in particular,

$$
\begin{aligned}
|\Phi(n ; T)|=\left|a_{n}\right| \cdot\left|B^{\prime}\left(\lambda_{m}\right)\right| \leqq & M(T) \cdot T^{-n}, \\
& n=\lambda_{m}, \quad m=1,2, \cdots,
\end{aligned}
$$

valid for every $T$ in $0<T<T_{0}$.

This formula is the basis of our results: The growth of $f(x), x>0$, reflects in $M(T)$, assumptions on the gap exponents $\lambda_{k}$ enter into $\left|B^{\prime}\left(\lambda_{m}\right)\right|$, and combining both we obtain information about $\left|a_{n}\right|$.

By way of an example, if $T_{0}=\infty$ and $f(x)$ is bounded for $x>0$, we have $M(T)=O(\log T), T \rightarrow+\infty$, and we see that the right-hand side of (1.11) tends to zero for $T \rightarrow+\infty$ and every fixed $n>0$. Since $B^{\prime}\left(\lambda_{m}\right) \neq 0$ we get $a_{n}=0, n>0$; hence $f$ is constant; this is Macintyre's result mentioned above.

2. On the derivative of Blaschke products. Let $\lambda=\left\{\lambda_{k}\right\}$ be a sequence of positive numbers, $0<\lambda_{1}<\lambda_{2}<\cdots$, with

$$
\begin{gathered}
\lambda_{k+1}-\lambda_{k} \geqq \delta>0, \\
\sum_{k=1}^{\infty} \frac{1}{\lambda_{k}}<\infty .
\end{gathered}
$$

In order to estimate $\left|a_{n}\right|$ by (1.11), it is necessary to obtain information about 


$$
\left|B^{\prime}\left(\lambda_{m}\right)\right|^{-1}=2 \lambda_{m} p_{m} \quad \text { with } \quad p_{m}=\prod_{k \neq m}\left|\frac{\lambda_{k}+\lambda_{m}}{\lambda_{k}-\lambda_{m}}\right| .
$$

We see that $p_{m}>1$ for all $m$, and in $\S 2$ we shall discuss estimates of $p=\left\{p_{m}\right\}$ from above for various choices of $\lambda$.

\subsection{The general case.}

Theorem 1. If $\lambda$ satisfies (2.1), we have

$$
0<\log p_{m}=o\left(\lambda_{m}\right), \quad m \rightarrow \infty .
$$

Proof. We write $p_{m}=\Pi_{1} \Pi_{2} \Pi_{3}$, where $\Pi_{1}$ contains the factors with $k<m, \Pi_{2}$ those with $\lambda_{m}<\lambda_{k}<2 \lambda_{m}$, and $\Pi_{3}$ those with $\lambda_{k} \geqq 2 \lambda_{m}$. For $\Pi_{1}$ we have $\lambda_{m}-\lambda_{k} \geqq(m-k) \delta, k=1,2, \cdots, m-1$, and therefore

$$
\Pi_{1}=\prod_{k<m} \frac{\lambda_{m}+\lambda_{k}}{\lambda_{m}-\lambda_{k}} \leqq\left(\frac{2 \lambda_{m}}{\delta}\right)^{m-1} \cdot \frac{1}{(m-1) !} \leqq\left(\frac{2 \lambda_{m} e}{\delta(m-1)}\right)^{m-1},
$$

since $n^{n} / n ! \leqq e^{n}, n=0,1,2, \cdots$. This implies

$$
\log \Pi_{1} \leqq \lambda_{m} \cdot \frac{m-1}{\lambda_{m}}\left[\log \frac{\lambda_{m}}{m-1}+C\right]=o\left(\lambda_{m}\right), \quad m \rightarrow \infty,
$$

since $\lambda_{m} / m \rightarrow \infty$ which is a consequence of $(2.1 \mathrm{~b})$.

Assume $\Pi_{2}$ contains $N$ factors (if $N=0$, put $\Pi_{2}=1$ ). Then as above

and hence

$$
\Pi_{2} \leqq\left(\frac{3 \lambda_{m}}{\delta}\right)^{N} \cdot \frac{1}{N !} \leqq\left(\frac{3 \lambda_{m} e}{\delta N}\right)^{N}
$$

$$
\log \Pi_{2} \leqq \lambda_{m} \cdot \frac{N}{\lambda_{m}}\left[\log \frac{\lambda_{m}}{N}+C\right]=o\left(\lambda_{m}\right), \quad m \rightarrow \infty,
$$

since $m+N=o\left(\lambda_{m+N}\right)=o\left(\lambda_{m}\right)$, hence $N=o\left(\lambda_{m}\right)$ or $\lambda_{m} / N \rightarrow \infty$.

In $\Pi_{3}$ we finally have $\lambda_{k} \geqq 2 \lambda_{m}$ or $\left(\lambda_{k}-\lambda_{m}\right)^{-1} \leqq 2 / \lambda_{k}$, so that

$$
\frac{\lambda_{k}+\lambda_{m}}{\lambda_{k}-\lambda_{m}}=1+\frac{2 \lambda_{m}}{\lambda_{k}-\lambda_{m}} \leqq 1+4 \frac{\lambda_{m}}{\lambda_{k}}
$$

and therefore

$$
\log \Pi_{3} \leqq \sum \log \left(1+4 \frac{\lambda_{m}}{\lambda_{k}}\right)<4 \lambda_{m} \cdot \sum_{\lambda_{k} \geqq \lambda_{m}} \frac{1}{\lambda_{k}}=o\left(\lambda_{m}\right), \quad m \rightarrow \infty .
$$

Combining our results, we arrive at (2.3).

2.2. Hadamard sequences. The sequence $\lambda=\left\{\lambda_{k}\right\}$ is called a Hadamard sequence if

$$
\lambda_{k+1} / \lambda_{k} \geqq \theta, \quad k=1,2, \cdots, \quad \text { for some } \theta>1 .
$$


Theorem 2. The sequence $p=\left\{p_{m}\right\}$ is bounded if and only if $\lambda$ is a Hadamard sequence. If $\lambda_{k+1} / \lambda_{k} \rightarrow \infty, k \rightarrow \infty$, then $p_{m} \rightarrow 1, m \rightarrow \infty$.

Proof. First let $\lambda$ be a Hadamard sequence. We write $p_{m}=\Pi_{1} \Pi_{2}$, where

$$
\Pi_{1}=\prod_{k<m} \frac{\lambda_{m}+\lambda_{k}}{\lambda_{m}-\lambda_{k}}=\prod_{j=1}^{m-1} \frac{1+\lambda_{m-\jmath} / \lambda_{m}}{1-\lambda_{m-j} / \lambda_{m}} \leqq \prod_{j=1}^{m-1} \frac{1+\theta^{-j}}{1-\theta^{-j}}<\prod_{j=1}^{\infty} \frac{1+\theta^{-j}}{1-\theta^{-j}},
$$

since $\lambda_{m-j} / \lambda_{m} \leqq \theta^{-j}, j=1,2, \cdots, m-1$. Similarly,

$$
\Pi_{2}=\prod_{k>m} \frac{\lambda_{k}+\lambda_{m}}{\lambda_{k}-\lambda_{m}}=\prod_{j=1}^{\infty} \frac{1+\lambda_{m} / \lambda_{m+j}}{1-\lambda_{m} / \lambda_{m+j}} \leqq \prod_{j=1}^{\infty} \frac{1+\theta^{-j}}{1-\theta^{-j}},
$$

and therefore

$$
1<p_{m} \leqq C(\theta), \quad m=1,2, \cdots .
$$

If $\lambda$ is not a Hadamard sequence, there exists a sequence of indices $k$ for which $\lambda_{k+1} / \lambda_{k} \rightarrow 1$. Since every factor in $p_{m}$ is greater than 1 , we have for these indices

$$
p_{k}>\frac{\lambda_{k+1}+\lambda_{k}}{\lambda_{k+1}-\lambda_{k}}=\frac{\lambda_{k+1} / \lambda_{k}+1}{\lambda_{k+1} / \lambda_{k}-1} \rightarrow \infty .
$$

Now let $\lambda_{k+1} / \lambda_{k} \rightarrow \infty, k \rightarrow \infty$, so that for given $\epsilon, 0<\epsilon \leqq 1 / 2$, there exists $N=N(\epsilon)$ such that $\lambda_{k-1} / \lambda_{k} \leqq \epsilon, k>N$. Observing

$$
\log \frac{1+x}{1-x} \leqq 3 x \text { in } 0 \leqq x \leqq \frac{1}{2},
$$

we get for $m>N$,

$$
\log \prod_{k>m} \frac{\lambda_{k}+\lambda_{m}}{\lambda_{k}-\lambda_{m}}=\sum_{k>m} \log \frac{1+\lambda_{m} / \lambda_{k}}{1-\lambda_{m} / \lambda_{k}} \leqq 3 \sum_{j=1}^{\infty} \frac{\lambda_{m}}{\lambda_{m+j}} \leqq 3 \frac{\epsilon}{1-\epsilon},
$$

since $\lambda_{m} / \lambda_{m+j} \leqq \epsilon^{j}, j=1,2, \cdots$. On the other hand,

$$
\log \prod_{k<m} \frac{\lambda_{m}+\lambda_{k}}{\lambda_{m}-\lambda_{k}}=\sum_{k<m} \log \frac{1+\lambda_{k} / \lambda_{m}}{1-\lambda_{k} / \lambda_{m}} \leqq 3 \sum_{k<m} \frac{\lambda_{k}}{\lambda_{m}}=3 \sum_{k<N}+3 \sum_{N \leqq k<m} ;
$$

notice that $\lambda_{k} / \lambda_{m} \leqq \lambda_{m-1} / \lambda_{m} \leqq \epsilon \leqq 1 / 2$. For $N \leqq k<m$ we have

$$
\lambda_{k} \leqq \epsilon^{j} \lambda_{k+j} \text {, i.e., } \lambda_{k} \leqq \epsilon^{m-k} \lambda_{m},
$$

so that the last sum is equal to or less than

$$
\sum_{N \leqq k<m} \epsilon^{m-k}<\frac{\epsilon}{1-\epsilon},
$$

whereas $\sum_{k<N}<\epsilon$ for $m$ large enough. This proves $\log p_{m} \rightarrow 0, m \rightarrow \infty$.

2.3. The case $\lambda_{k}=k^{\alpha}, \alpha>1$. We shall need the following result. 
Lemma 2. For every $\alpha>1$,

$$
J(\alpha)=\int_{0}^{\infty} \log \left|\frac{x^{\alpha}+1}{x^{\alpha}-1}\right| d x=\pi \tan \frac{\pi}{2 \alpha} .
$$

Proof. Let $C$ be the path consisting of

$$
\begin{gathered}
0 \leqq x \leqq R ; \quad|z|=R, \quad 0 \leqq \arg z \leqq \frac{\pi}{2 \alpha} ; \\
z=r e^{i \pi /(2 \alpha)}, \quad 0 \leqq r \leqq R,
\end{gathered}
$$

indented by circular arcs of radii $\rho$ around $z=0$ and $z=1$. Then

$$
\int_{C} \log \frac{z^{\alpha}+1}{z^{\alpha}-1} d z=0 .
$$

Letting $\rho \rightarrow 0, R \rightarrow \infty$, and separating real and imaginary parts, we arrive at $(2.5)$.

Now we shall study the sequence $p=\left\{p_{m}\right\}$ in the case $\lambda_{k}=k^{\alpha}, \alpha>1$.

Theorem 3. If $\lambda_{k}=k^{\alpha}, k=1,2, \cdots, \alpha>1$, then

$$
0<\log p_{m}<J(\alpha) m=J(\alpha) \lambda_{m}^{1 / \alpha}, \quad m=1,2, \cdots,
$$

where $J(\alpha)$ is defined in (2.5). The constant $J(\alpha)$ is best possible.

Proof. We have for all $m=1,2, \cdots$,

$$
\frac{1}{m} \log p_{m}=\frac{1}{m} \sum_{k \neq m} \log \left|\frac{\lambda_{k} / \lambda_{m}+1}{\lambda_{k} / \lambda_{m}-1}\right|=\frac{1}{m} \sum_{k \neq m} \log \left|\frac{(k / m)^{\alpha}+1}{(k / m)^{\alpha}-1}\right|,
$$

which can be interpreted às the lower Riemann sum for the function

$$
h(x)=\log \left|\frac{x^{\alpha}+1}{x^{\alpha}-1}\right|, \quad 0 \leqq x<\infty,
$$

and $\Delta x=1 / m$. This implies (2.6).

That actually $m^{-1} \cdot \log p_{m} \rightarrow J(\alpha), m \rightarrow \infty$, follows from the fact that the Riemann sums are integrals over step functions $h_{m}(x)$ for which

$$
h_{m}(x) \rightarrow h(x), m \rightarrow \infty, 0 \leqq x<\infty \text {, and } h_{m}(x) \leqq h(x) .
$$

The Lebesgue convergence theorem asserts that

$$
\frac{1}{m} \log p_{m}=\int_{0}^{\infty} h_{m}(x) d x \rightarrow \int_{0}^{\infty} h(x) d x=J(\alpha), \quad m \rightarrow \infty .
$$

Remark. Since $h(x)$ is monotonic and convex in each of the intervals $(0,1)$ and $(1, \infty)$, it is easy to see that even

$$
m^{-1} \log p_{m} \nearrow J(\alpha),
$$$$
m \nearrow \infty .
$$

Now we ask ourselves whether (2.6) still holds if $\lambda_{k}=k^{\alpha}$ is replaced by 
$\lambda_{k} \cong k^{\alpha}$. The sequence $m^{-1} \log p_{m}$ may be unbounded if only $\lambda_{k} \cong k^{\alpha}, k \rightarrow \infty$, is assumed. We show this for $\alpha=2$, defining $\left\{\lambda_{k}\right\}$ from a certain index on as blocks of consecutive integers. In the $q$ th block

$$
\lambda_{k_{q}}={k_{q}}^{2} \text { and } \quad \lambda_{k_{q}+j}={k_{q}}^{2}+j, \quad 0<j \leqq j(q)=\left[\frac{k_{q}}{\log \log k_{q}}\right] .
$$

If $k_{q+1}=k_{q}+j(q)+1$, we have $k_{q+1}^{2}>k_{q}^{2}+j(q)+1$, so that the $q$-block and the $(q+1)$-block do not overlap. We now have

$$
\frac{\lambda_{k}}{k^{2}}=\frac{\lambda_{k_{q}+j}}{\left(k_{q}+j\right)^{2}}=\frac{k_{q}{ }^{2}+j}{\left(k_{q}+j\right)^{2}}=\frac{1+j / k_{q}{ }^{2}}{\left(1+j / k_{q}\right)^{2}} \rightarrow 1, \quad k \rightarrow \infty,
$$

and on the other hand, for $m=k_{q}$,

$$
p_{m}=\prod_{k \neq m}\left|\frac{\lambda_{k}+\lambda_{m}}{\lambda_{k}-\lambda_{m}}\right| \geqq \prod_{j=1}^{j(q)} \frac{\lambda_{m+j}+\lambda_{m}}{\lambda_{m+j}-\lambda_{m}} \geqq \frac{\lambda_{m}^{j(q)}}{j(q) !}=k_{q}^{j(q)} \cdot \frac{k_{q}^{j(q)}}{j(q) !} \geqq k_{q}^{j(q)},
$$

since $k_{q} \geqq j(q)$. Therefore

$$
\frac{1}{m} \log p_{m} \geqq \frac{1}{k_{q}} j(q) \log k_{q} \cong \frac{\log k_{q}}{\log \log k_{q}} \rightarrow \infty, \quad q \rightarrow \infty .
$$

2.4. The case $\lambda_{k+1}-\lambda_{k} \geqq \theta \lambda_{k}{ }^{\sigma}, 0<\sigma<1, \theta>0$. We first remark that this condition implies

$$
\lambda_{k+1}^{\tau}-\lambda_{k}^{\tau} \geqq A=A(\theta, \sigma)>0 \text { with } \tau=1-\sigma, \text { for } k=1,2, \cdots .
$$

Because we have

$$
\lambda_{k+1}^{\tau} \geqq \lambda_{k}{ }^{\tau}\left(1+\frac{\theta}{\lambda_{k}^{1-\sigma}}\right)^{\tau} \geqq \lambda_{k}^{\tau}\left(1+\frac{\tau \theta}{2} \lambda_{k}^{\sigma-1}\right), \quad k>k_{0}(\sigma, \theta),
$$

so that $\lambda_{k+1}^{\tau}-\lambda_{k}^{\tau} \geqq \tau \theta / 2, k>k_{0}(\sigma, \theta)$, which implies (2.7).

Theorem 4. If $\lambda_{k+1}-\lambda_{k} \geqq \theta \lambda_{k}{ }^{\sigma}$ for $0<\sigma<1$ and some $\theta>0$, then

$$
0<\log p_{m}<\frac{J\left(\tau^{-1}\right)}{A} \cdot \lambda_{m}{ }^{\tau}, \quad m=1,2, \cdots,
$$

where $\tau=1-\sigma, A$ is the constant in (2.7), and $J$ is the integral defined in $(2.5)$.

Remark. In the special case $\lambda_{k+1}-\lambda_{k} \geqq \theta \sqrt{\lambda_{k}}$ we therefore obtain

$$
\log p_{m}=O\left(\sqrt{\lambda_{m}}\right), \quad m \rightarrow \infty .
$$

If $\lambda_{k}=k^{\alpha}, \alpha>1$, we have $\lambda_{k+1}-\lambda_{k} \sim k^{\alpha-1}=\lambda_{k}^{1-1 / \alpha}$, and (2.8) gives

$$
\log p_{m}=O\left({\lambda_{m}}^{1 / \alpha}\right), \quad m \rightarrow \infty,
$$

as already seen in (2.6).

Proof. Notice that $\lambda_{k} \geqq($ const. $) \cdot k^{1+\sigma}$, so that $\sum \lambda_{k}^{-1}<\infty$. Now 


$$
\begin{aligned}
\log p_{m} & =\sum_{k \neq m}\left|\frac{\lambda_{k} / \lambda_{m}+1}{\lambda_{k} / \lambda_{m}-1}\right| \\
& =\sum_{k<m} \log \left|\frac{x_{k}^{\gamma}+1}{x_{k}^{\gamma}-1}\right|+\sum_{k>m} \log \left|\frac{x_{k}^{\gamma}+1}{x_{k}^{\gamma}-1}\right|,
\end{aligned}
$$

where we put

$$
x_{k}=x_{k}^{(m)}=\left(\frac{\lambda_{k}}{\lambda_{m}}\right)^{\tau}, \quad k=1,2, \cdots, \text { and } \gamma=\tau^{-1}>1 .
$$

The distance between two consecutive $x_{k}^{(m)}$ is

$$
x_{k+1}^{(m)}-x_{k}^{(m)}=\lambda_{m}{ }^{-\tau}\left(\lambda_{k+1}^{\tau}-\lambda_{k}^{\tau}\right) \geqq A \lambda_{m}{ }^{-\tau}, \quad k=1,2, \cdots,
$$

because of (2.7). Combining this with (2.10) we obtain

$$
\begin{aligned}
A \lambda_{m}{ }^{-\tau} \log p_{m} \leqq \sum_{k<m} \log \left|\frac{x_{k}^{\gamma}+1}{x_{k}{ }^{\gamma}-1}\right| & \left(x_{k+1}-x_{k}\right) \\
& +\sum_{k>m} \log \left|\frac{x_{k}^{\gamma}+1}{x_{k}^{\gamma}-1}\right|\left(x_{k}-x_{k-1}\right),
\end{aligned}
$$

and this may again be interpreted as the lower Riemann sum for the function $h(x)$ of $\S 2.3$ (with $\alpha=\gamma$ ) and the division points $x_{k}=x_{k}{ }^{(m)}$, $k=1,2, \cdots$. Therefore the right-hand side of the last inequality is less than $J(\gamma)=J\left(\tau^{-1}\right)$, as stated in (2.8).

2.5. Generalization in the case of Hadamard sequences. Let $\lambda=\left\{\lambda_{k}\right\}$ be a Hadamard sequence, $\lambda_{k+1} / \lambda_{k} \geqq \theta>1$. In a generalization of our method outlined in $\S 1.3$ we shall need information about the sequence $q=\left\{q_{m}\right\}$ defined by

$$
q_{m}=\prod_{k \neq m}\left|\frac{\lambda_{k}+\lambda_{m}+2 \gamma_{m}}{\lambda_{k}-\lambda_{m}}\right|, \quad \text { where } \quad \gamma_{m}=\frac{\lambda_{m}}{m} .
$$

Theorem 5. If $\lambda$ is a Hadamard sequence, $q=\left\{q_{m}\right\}$ is bounded.

Proof. Since $\lambda_{m} \leqq \theta^{-j} \lambda_{m+j}, j=1,2, \cdots$, we have

$$
\prod_{k>m} \leqq \prod_{k>m} \frac{1+3 \lambda_{m} / \lambda_{k}}{1-\lambda_{m} / \lambda_{k}} \leqq \prod_{j=1}^{\infty} \frac{1+3 \theta^{-j}}{1-\theta^{-j}} .
$$

Furthermore

and finally

$$
\prod_{k<m}\left(1-\lambda_{k} / \lambda_{m}\right) \geqq \prod_{j=1}^{\infty}\left(1-\theta^{-j}\right) \neq 0,
$$

$$
\prod_{k<m}\left(1+\frac{\lambda_{k}+2 \gamma_{m}}{\lambda_{m}}\right)<\exp \left\{\sum_{k<m} \frac{\lambda_{k}}{\lambda_{m}}+\frac{2 \gamma_{m}}{\lambda_{m}} \cdot m\right\}<\exp \left\{\sum_{j=1}^{\infty} \theta^{-j}+2\right\} ;
$$

note $1+x<e^{x}$. Therefore $\left\{q_{m}\right\}$ is bounded. 


\section{Application of general method to entire functions.}

3.1. The general gap condition. First we assume that an entire function $f(z)=\sum a_{n} z^{n}$ is given, the coefficients of which satisfy the general gap condition

$$
a_{n}=0 \text { for } n \neq \lambda_{k}, \quad k=1,2, \cdots, \text { where } \sum_{k=1}^{\infty} \frac{1}{\lambda_{k}}<\infty ;
$$

here the $\lambda_{k}$ are integers with $0<\lambda_{1}<\lambda_{2}<\cdots$, so that in particular $a_{0}=0$. Using (1.11) and (2.2) we obtain the coefficient estimate

$$
\left|a_{n}\right| \leqq 2 n p_{m} \cdot M(T) \cdot T^{-n}, \quad n=\lambda_{m}, m=1,2, \cdots,
$$

where $\left\{p_{m}\right\}$ is the sequence studied in $\S 2$, and where

$$
M(T)=\int_{0}^{T} \frac{|f(t)|}{t} d t, \quad 0<T<\infty .
$$

Note that (3.2) is valid for every $T$ in $0<T<\infty$.

If $f$ is of polynomial growth on the positive axis, $f(x)=O\left(x^{\alpha}\right), x \rightarrow+\infty$, $\alpha>0$, we get $M(T)=O\left(T^{\alpha}\right), T \rightarrow \infty$, so that the right-hand side of (3.2) tends to zero for $T \rightarrow \infty$ and every fixed $n>\alpha$. Hence $a_{n}=0$, $n>\alpha$, i.e., $f$ is a polynomial of degree $\leqq \alpha$.

In the more interesting case of exponential growth

$$
f(x)=O\left(e^{x^{\alpha}}\right), \quad x \rightarrow+\infty, \quad \alpha>0,
$$

we have

$$
\begin{aligned}
\int_{1}^{T} e^{t^{\alpha}} \frac{d t}{t}= & \frac{1}{\alpha} \int_{1}^{T^{\alpha}} e^{u} \frac{d u}{u}<\frac{2}{\alpha} \int_{T^{\alpha} / 2}^{T^{\alpha}} e^{u} \frac{d u}{u} \\
& <\frac{4}{\alpha T^{\alpha}} \int_{0}^{T^{\alpha}} e^{u} d u<\frac{4}{\alpha} \frac{e^{T^{\alpha}}}{T^{\alpha}}
\end{aligned}
$$

and therefore

$$
M(T)=O\left(\frac{e^{T^{\alpha}}}{T^{\alpha}}\right), \quad T \rightarrow \infty .
$$

If we choose $T>0$ so that $T^{\alpha}=1+n / \alpha$, we obtain

$$
\begin{aligned}
M(T) T^{-n} & =O(1) e^{1+n / \alpha}\left(1+\frac{n}{\alpha}\right)^{-(1+n / \alpha)} \\
& =O(1) \cdot\left[\sqrt{n} \Gamma\left(1+\frac{n}{\alpha}\right)\right]^{-1}
\end{aligned}
$$

by Stirling's formula, and (3.2) yields 


$$
a_{n}=O\left(\frac{\sqrt{n}}{\Gamma\left(1+\frac{n}{\alpha}\right)}\right) \cdot p_{m}, \quad n=\lambda_{m} \rightarrow \infty .
$$

Combining this with Theorem 1 , we obtain: ${ }^{1}$

Theorem 6. If $f(z)=\sum_{n=0}^{\infty} a_{n} z^{n}$ is an entire function satisfying the gap condition (3.1) and if $f(x)=O\left(e^{x^{\alpha}}\right), x \rightarrow+\infty, \alpha>0$, then

$$
a_{n}=O(1) \frac{e^{\epsilon n}}{\Gamma\left(1+\frac{n}{\alpha}\right)}, n \rightarrow \infty,
$$

for every $\epsilon>0$. In particular, $f$ is at most of type 1 of order $\alpha$.

The last statement is proved by writing

$$
|f(z)| \leqq \sum_{n=0}^{\infty}\left|a_{n}\right| \cdot|z|^{n}=O(1) \sum_{n=0}^{\infty} \frac{y^{n}}{\Gamma\left(1+\frac{n}{\alpha}\right)} \text { with } y=e^{\epsilon}|z|,
$$

and observing that Mittag-Leffler's function

$$
E_{\gamma}(y)=\sum_{n=0}^{\infty} \frac{y^{n}}{\Gamma(1+\gamma n)}=O\left(\exp y^{1 / \gamma}\right), \quad y \rightarrow+\infty, \gamma>0,
$$

(see, for instance, [6, p. 198]). Thus

$$
|f(z)|=O\left[\exp \left(e^{\epsilon \alpha}|z|^{\alpha}\right)\right], \quad|z| \rightarrow \infty,
$$

for every $\epsilon>0$, and the result follows.

The case $\alpha=1$ is of particular importance in the theory of Borel summability. If $B-\sum_{n=0}^{\infty} c_{n}=s$, one easily finds that

$$
\sum_{n=0}^{\infty} \frac{c_{n} x^{n}}{n !}=o\left(e^{x}\right), \quad x \rightarrow+\infty,
$$

so that by Theorem 6 , with $\alpha=1$,

$$
c_{n}=O\left(e^{\epsilon n}\right), \quad n \rightarrow \infty,
$$

for every $\epsilon>0$, provided the $c_{n}$ satisfy the gap hypothesis (3.1).

1 Professor Korevaar pointed out to me that Theorem 6 is closely related to work on the Müntz-Szasz approximation theorem done by Clarkson-Erdös [3] and Korevaar [7]. The first authors proved ([3, pp. 6-7], see also [7, p. 756]):

$$
\text { inf }\left\|x^{\lambda_{n}}-P(x)\right\| \geqq(1+\epsilon)^{-\lambda_{n}}, \quad \epsilon>0, \quad n>n_{0}(\epsilon),
$$

where \|\| is the $L_{2}$ norm in $(0,1)$, and where the infimum ranges over all $P(x)$ $=\sum_{k \neq n} \alpha_{k} x^{\lambda_{k}}$. It is easy to see that $(*)$ furnishes another proof of Theorem 6 . 
Theorem 7. If $\sum_{n=0}^{\infty} c_{n}$ is Borel summable and satisfies the gap condition (3.1), the power series $\sum_{n=0}^{\infty} c_{n} z^{n}$ will converge in $|z|<1$.

If the gap condition (3.1) is strengthened to

$$
c_{n}=0 \text { for } n \neq \lambda_{k}, k=1,2, \cdots, \text { where } \lambda_{k+1}-\lambda_{k} \geqq \theta \sqrt{\lambda_{k}}, \theta>0,
$$

we may apply a result of Meyer-König and Zeller [10, p. 205] to obtain the convergence of $\sum_{n=0}^{\infty} c_{n}$ from its Borel summability. This is the unrestricted high indices theorem for Borel summability proved earlier by the author [5].

3.2. Precision of the gap condition in Theorem 6. We shall now see that the gap condition (3.1) is best possible in Theorem 6 .

Theorem 8. For every sequence $\left\{\lambda_{k}\right\}$ of integers with

$$
0<\lambda_{1}<\lambda_{2}<\cdots \text { and } \sum_{k=1}^{\infty} \lambda_{k}^{-1}=\infty
$$

there exists an entire function $f(z)=\sum_{n=0}^{\infty} a_{n} z^{n}$ with $a_{n}=0$ for $n \neq \lambda_{k}$, which is $O(1)$ for $z=x \rightarrow+\infty$, but of infinite order.

This result is essentially due to Macintyre. We need:

Lemma 3. If $\left\{\lambda_{k}\right\}$ is a sequence of integers for which (3.5) holds, there exists a subsequence $\left\{\lambda_{k_{m}}\right\}$ such that

$$
\begin{aligned}
& \text { (a) } \sum_{m=1}^{\infty} \lambda_{k_{m}}^{-1}=\infty, \\
& \text { (b) } \lambda_{k_{m}} / m \rightarrow \infty, m \rightarrow \infty .
\end{aligned}
$$

Proof. Let $\left\{\epsilon_{k}\right\}$ be a monotonic null sequence for which $\sum_{k=1}^{\infty} \epsilon_{k} \lambda_{k}{ }^{-1}=\infty$; we may assume $\epsilon_{1} \leqq 1$. Put $j_{m}=\left[\epsilon_{m}{ }^{-1}\right], m=1,2, \cdots$, so that $1 \leqq j_{m} \nearrow \infty$, and put

$$
k_{1}=1, k_{m+1}=k_{m}+j_{m}, m \geqq 1 \text {, so that } k_{m}=1+\sum_{\nu=1}^{m-1} j_{\nu}, m \geqq 1 .
$$

Thereby the subsequence is determined, and we claim (3.6) to be fulfilled.

First, we have for monotony reasons

$$
\epsilon_{k_{m}} \lambda_{k_{m}}^{-1} \geqq \epsilon_{k} \lambda_{k}^{-1} \text { in } \quad i_{m}: k_{m} \leqq k<k_{m+1},
$$

therefore

$$
j_{m} \epsilon_{k_{m}} \lambda_{k_{m}}^{-1} \geqq \sum_{i_{m}} \epsilon_{k} \lambda_{k}^{-1}, \quad m=1,2, \cdots,
$$

and if we take the sum over $m$ and observe $j_{m} \leqq j_{k_{m}} \leqq \epsilon_{k_{m}}^{-1}$, we get

$$
\sum_{m} \lambda_{k_{m}}^{-1} \geqq \sum_{k} \epsilon_{k} \lambda_{k}^{-1}=\infty \text {. }
$$


Property (3.6b) follows from

$$
\frac{\lambda_{k_{m}}}{m}=\frac{\lambda_{k_{m}}}{k_{m}} \cdot \frac{k_{m}}{m} \geq 1 \cdot \frac{k_{m}}{m}>\frac{1}{m} \sum_{\nu=1}^{m-1} j_{\nu} \rightarrow \infty, \quad m \rightarrow \infty .
$$

Proof of Theorem 8. Extract from $\left\{\lambda_{k}\right\}$ a subsequence $\left\{\lambda_{k_{m}}\right\}$ satisfying (3.6). According to Macintyre [9, pp. 287-290] there exists an entire transcendental function $f(z)=\sum_{n=0}^{\infty} a_{n} z^{n}$ with $a_{n}=0$ for $n \neq \lambda_{k_{m}}$, $m=1,2, \cdots$, hence certainly $a_{n}=0$ for $n \neq \lambda_{k}, k=1,2, \cdots$, which is bounded for $z=x>0$. Property (3.6b) shows that $f$ has Fabry gaps, and by a result of Pólya [11, p. 631] $f$ cannot be of finite order.

3.3. Entire functions with Hadamard gaps. We now consider the case that $f(z)=\sum_{n=0}^{\infty} a_{n} z^{n}$ with

(3.7) $\quad a_{n}=0$ for $n \neq \lambda_{k}, k=1,2, \cdots$, where $\lambda_{k+1} / \lambda_{k} \geqq \theta>1$.

Lemma 4. If $\left\{n_{k}\right\}$ is a sequence of natural numbers with $n_{k+1} / n_{k} \geqq \theta>1$, then

$$
g(x)=\sum_{k=1}^{\infty} \frac{\sqrt{n_{k}}}{n_{k} !} x^{n_{k}}=O\left(e^{x}\right), \quad x \rightarrow+\infty .
$$

Proof. Let $\gamma>0$ be so small that $(1+\gamma) /(1-\gamma)<\theta$. Given $x>0$, there is at most one of the numbers $n_{k}$ in the interval $(1-\gamma) x,(1+\gamma) x$; otherwise $n_{k+1} / n_{k} \leqq(1+\gamma) /(1-\gamma)<\theta$ for some $k$. Assuming that $n_{k^{\prime}}$ is the integer in that interval, we have

$$
e^{-x} g(x)=\sum e^{-x} \frac{\sqrt{n_{k}}}{n_{k} !} x^{n_{k}}+e^{-x} \frac{\sqrt{n_{k^{\prime}}}}{n_{k^{\prime}} !} x^{n_{k^{\prime}}},
$$

where the sum ranges over $n_{k}$ with $\left|n_{k}-x\right|>\gamma x$. This sum is therefore less than

$$
\sum_{|n-x|>\gamma x} e^{-x} \frac{\sqrt{n}}{n !} x^{n}=o(1), x \rightarrow+\infty,
$$

(see [6, pp. 200-201]). Moreover, for all $n=0,1,2, \cdots$ and $x \geqq 0$,

$$
e^{-x} \frac{\sqrt{n}}{n !} x^{n} \leq e^{-n} \frac{\sqrt{n}}{n !} n^{n} \leq C,
$$

by Stirling's formula. This proves (3.8).

Theorem 9. If $f(z)=\sum_{n=0}^{\infty} a_{n} z^{n}$ is an entire function satisfying the gap condition (3.7), and $f(x)=O\left(e^{x}\right), x \rightarrow+\infty$, then

$$
a_{n}=O(1) \frac{\sqrt{n}}{n !}, \quad n \rightarrow \infty,
$$

and $O(1)$ cannot be replaced by $o(1)$. Furthermore $f(z)=O\left(e^{|z|}\right),|z| \rightarrow \infty$. 
Proof. The estimate of $a_{n}$ follows from (3.3) and Theorem 2. Lemma 4 shows that $O(1)$ cannot be replaced by $o(1)$, and that

$$
|f(z)| \leq \sum_{n=0}^{\infty}\left|a_{n}\right| \cdot|z|^{n}=O(1) \cdot \sum_{k=1}^{\infty} \frac{\sqrt{\lambda_{k}}}{\lambda_{k} !}|z|^{\lambda_{k}}=O\left(e^{|z|}\right), \quad|z| \rightarrow \infty .
$$

4. On the coefficients of Dirichlet series. We shall now derive results similar to those of $\S 3$, but for the coefficients of Dirichlet series the growth of which is known as $z$ approaches the boundary of convergence. We assume that

$$
f(z)=\sum_{n=1}^{\infty} a_{n} e^{-\lambda_{n} z}
$$

converges for $\operatorname{Re} z=x>0$, and that

$$
0<\lambda_{1}<\lambda_{2}<\cdots, \quad \lambda_{n+1}-\lambda_{n} \geqq \delta>0, \quad \sum_{n=1}^{\infty} \frac{1}{\lambda_{n}}<\infty .
$$

The second of these conditions implies that (4.1) converges absolutely in $x>0$ (see, for example, $[1$, p. 4]).

4.1. Modification of general method. Instead of (1.4) we start from the transformation

$$
H(z ; T)=\int_{T}^{\infty} f(t) e^{t z} d t, \quad z=x+i y,
$$

for fixed $T>0$. Since $f(t)=O\left(e^{-\lambda_{1} t}\right), t \rightarrow+\infty$, the integral converges for $x<\lambda_{1}$ representing an analytic function in that halfplane. On $x=0$ we have

$$
|H(z ; T)| \leqq \int_{T}^{\infty}|f(t)| d t, \quad z=i y .
$$

The analytic continuation of $H(z ; T)$ beyond $\operatorname{Re} z=\lambda_{1}$ can be obtained by inserting (4.1) into (4.3) and reversing the order of integration and summation for $\operatorname{Re} z<0$. We obtain

$$
H(z ; T)=-e^{T z} \cdot \sum_{n=1}^{\infty} \frac{a_{n}}{z-\lambda_{n}} e^{-T \lambda_{n}}, \quad \operatorname{Re} z<0 .
$$

However, since $\sum_{n=1}^{\infty}\left|a_{n}\right| e^{-T \lambda_{n}}<\infty$, the series in (4.5) converges uniformly for all $z$ with $\left|z-\lambda_{n}\right| \geqq \eta>0$, so that $H(z ; T)$ is meromorphic with simple poles at $z=\lambda_{n}$, at which $H(z ; T)$ has residues $-a_{n}$, $n=1,2, \cdots$. The poles are removed by considering

$$
\Phi(z ; T)=B(z) \cdot H(z ; T)
$$

with the Blaschke product $B(z)$ of (1.8), and it is found in the same way 
as in $\$ 1.3$ that

$$
|\Phi(z ; T)| \leqq e^{T x} \int_{T}^{\infty}|f(t)| d t, \quad \operatorname{Re} z=x \geqq 0 .
$$

Inserting $z=\lambda_{n}$ we obtain

and therefore

$$
\left|a_{n}\right| \cdot\left|B^{\prime}\left(\lambda_{n}\right)\right| \leqq e^{\lambda_{n} T} \int_{T}^{\infty}|f(t)| d t, \quad n=1,2, \cdots,
$$

$$
\left|a_{n}\right| \leqq 2 \lambda_{n} p_{n} \cdot e^{\lambda_{n} T} \int_{T}^{\infty}|f(t)| d t, \quad n=1,2, \cdots,
$$

for every $T>0$, where $\left\{p_{n}\right\}$ is the sequence defined in (2.2) and studied in $\$ 2$.

\subsection{Applications of (4.6) in special cases.}

(a) If $f \in L(0,1)$, let $T \rightarrow 0$ in (4.6).

Theorem 10. Let $\left\{\lambda_{n}\right\}$ satisfy (4.2), and let $f(z)=\sum_{n=1}^{\infty} a_{n} e^{-\lambda_{n} z}$ converge for $\operatorname{Re} z=x>0$. If $f \in L(0,1)$, we have

$$
\left|a_{n}\right| \leqq 2 \lambda_{n} p_{n} \int_{0}^{\infty}|f(t)| d t, \quad n=1,2, \cdots,
$$

where $\left\{p_{n}\right\}$ is the sequence studied in $\S 2$.

If we specialize $\lambda_{n}=n^{\alpha}, \alpha>1$, and use our results in $\S 2.3$, we obtain the estimate

$$
\left|a_{n}\right| \leqq 2 n^{\alpha} e^{J(\alpha) n} \int_{0}^{\infty}|f(t)| d t, \quad n=1,2, \cdots,
$$

with $J(\alpha)=\pi \tan (\pi / 2 \alpha)$. This includes a result of Kuttner [8, p. 124], according to which $\lim _{x \rightarrow 0+} f(x)=s$ implies $a_{n}=O\left(e^{\rho n}\right)$ for every $\rho>J(\alpha)$. His proof depends on other work of Miss Cartwright. Kuttner also notes that the estimate of $a_{n}$ is not true for $\rho<J(\alpha)$.

(b) If $f(t)=O(1 / t), t \rightarrow 0+$, we put $T=\lambda_{n}{ }^{-1}$ in (4.6) to obtain

$$
a_{n}=O\left(\lambda_{n} p_{n} \log \lambda_{n}\right), \quad n \rightarrow \infty .
$$

(c) If $f(t)=O\left(1 / t^{\beta}\right), t \rightarrow 0+, \beta>1$, we again put $T=\lambda_{n}{ }^{-1}$ in (4.6) to obtain

$$
a_{n}=O\left(\lambda_{n}{ }^{\beta} p_{n}\right), \quad n \rightarrow \infty .
$$

(d) If $f$ is of bounded variation in $(0,1)$, i.e., $f^{\prime} \in L(0,1)$, we get

$$
\left|a_{n}\right| \leqq 2 p_{n} \int_{0}^{\infty}\left|f^{\prime}(t)\right| d t, \quad n=1,2, \cdots .
$$

To see this, apply Theorem 10 to $f^{\prime}(z)=-\sum_{n=1}^{\infty} a_{n} \lambda_{n} e^{-\lambda_{n} z}$. 
4.3. Refinement of method in the case of Hadamard gaps. Our results of $\$ 4.2$ can be improved somewhat if $\left\{\lambda_{n}\right\}$ is a Hadamard sequence:

$$
\lambda_{n+1} / \lambda_{n} \geqq \theta, \quad n=1,2, \cdots, \quad \text { for some } \theta>1 .
$$

First we improve (4.6). To this end consider the function $H(z ; T)$ of (4.3) not in $\operatorname{Re} z \geqq 0$ but in $\operatorname{Re} z \geqq-\gamma$, where $\gamma \geqq 0$ is fixed for the moment. On $\operatorname{Re} z=-\gamma$ we have

$$
|H(z ; T)| \leqq \int_{T}^{\infty}|f(t)| e^{-\gamma t} d t, \quad z=-\gamma+i y .
$$

Instead of $B(z)$ we now use

$$
B(z ; \gamma)=\prod_{k=1}^{\infty} \frac{\lambda_{k}-z}{\lambda_{k}+z+2 \gamma} ;
$$

note that $|B(z ; \gamma)|=1$ on $\operatorname{Re} z=-\gamma$ and $|B(z ; \gamma)| \leqq 1$ in $\operatorname{Re} z \geqq-\gamma$. The function

$$
\Phi(z ; T ; \gamma)=B(z ; \gamma) \cdot H(z ; T), \quad \operatorname{Re} z \geqq-\gamma,
$$

is of exponential type in $\operatorname{Re} z \geqq-\gamma$ with $h_{\Phi}(0) \leqq T$, so that by Lemma 1 ,

$$
|\Phi(z ; T ; \gamma)| \leqq e^{T(x+\gamma)} \int_{T}^{\infty}|f(t)| e^{-\gamma t} d t, \quad \operatorname{Re} z=x \geqq-\gamma .
$$

Putting $z=\lambda_{n}$ we obtain

$$
\left|a_{n}\right| \cdot\left|B^{\prime}\left(\lambda_{n} ; \gamma\right)\right| \leqq e^{T\left(\lambda_{n}+\gamma\right)} \int_{T}^{\infty}|f(t)| e^{-\gamma t} d t, \quad n=1,2, \cdots,
$$

valid for all $\gamma \geqq 0, T>0$. In view of $\S 2.5$ we choose $\gamma=\gamma_{n}=\lambda_{n} / n$, and we obtain

$$
\left|a_{n}\right| \leqq 2\left(\lambda_{n}+\gamma_{n}\right) q_{n} \cdot e^{T\left(\lambda_{n}+\gamma_{n}\right)} \int_{T^{\prime}}^{\infty}|f(t)| e^{-\gamma_{n} t} d t, \quad n=1,2, \cdots,
$$

where $q_{n}$ are the numbers defined by (2.11) which are bounded if $\left\{\lambda_{n}\right\}$ is a Hadamard sequence (Theorem 5).

We now restrict the behavior of $f(t)$ as $t \rightarrow 0+$. Assume first that $f \in L(0,1)$; this always implies $f \in L(0, \infty)$ since $f(t)=O\left(e^{-\lambda_{1} t}\right), t \rightarrow \infty$. Letting $T \rightarrow 0$, (4.7) gives

$$
a_{n}=O\left(\lambda_{n}\right) \cdot \int_{0}^{\infty}|f(t)| e^{-\gamma_{n} t} d t, \quad n \rightarrow \infty,
$$

where, as always, $\gamma_{n}=\lambda_{n} / n$. If only $f \in L(0,1)$ is known we just get $a_{n}$ $=o\left(\lambda_{n}\right)$. If, however, $f \in L_{p}(0,1), p>1$, or if $f(t)=O\left(1 / t^{\beta}\right), t \rightarrow 0+$, $0<\beta<1$, we obtain by simple calculation 


$$
a_{n}=O\left(n^{1-\beta} \lambda_{n}^{\beta}\right)=O\left(\left(\log \lambda_{n}\right)^{1-\beta} \lambda_{n}{ }^{\beta}\right), \quad n \rightarrow \infty ;
$$

we have put $p=\beta^{-1}$ and observed that $n=O\left(\log \lambda_{n}\right)$.

In the case that $f(t)$ remains bounded as $t \rightarrow 0+$, our method gives $a_{n}=O(n)$ compared to the main step $a_{n}=O(1)$ in Ingham's proof of the high indices theorem (see [6, p. 173]). If, however, we assume slightly more, our method yields $\sum_{n=1}^{\infty}\left|a_{n}\right|<<_{-\lambda_{n}}^{\infty}$.

Theorem 11. Let $f(z)=\sum_{n=1}^{\infty} a_{n} e^{-\lambda_{n} z}$ with $\lambda_{n+1} / \lambda_{n} \geqq \theta>1$ converge for $\operatorname{Re} z=x>0$, and assume that

$$
f(x)=s+O\left(x^{\alpha}\right), \quad x \rightarrow 0+, \text { some } \alpha>0,
$$

or

$$
f^{\prime} \in L_{p}(0,1), \text { some } p>1 .
$$

Then $a_{n}=O\left(q^{n}\right)$ for some $q<1$; in particular, $\sum_{n=1}^{\infty}\left|a_{n}\right|^{\epsilon}<\infty$ for every $\epsilon>0$.

Again, our method does not give Zygmund's result: $\sum_{n=1}^{\infty}\left|a_{n}\right|<\infty$ if $f^{\prime} \in L(0,1)$ (see [13, p. 197]).

Proof. We may take $\alpha$ in $0<\alpha \leqq 1$, and then may assume $s=0$ in (4.10); otherwise consider $f^{*}(z)=f(z)-s e^{-\lambda_{1} z}$. Apply (4.8):

$$
a_{n}=O\left(n^{1+\alpha} \lambda_{n}{ }^{-\alpha}\right)=O\left(n^{1+\alpha} \theta^{-\alpha n}\right), \quad n \rightarrow \infty,
$$

since $\lambda_{n} \geqq \theta^{n-1} \lambda_{1}$. From this our conclusion follows.

If $(4.11)$ is assumed, an application of $(4.9)$, with $\beta=p^{-1}$, to $f^{\prime}(z)$ $=-\sum_{n=1}^{\infty} a_{n} \lambda_{n} e^{-\lambda_{n} z}$ yields

$$
a_{n}=O\left(n^{1-\beta} \lambda_{n}^{\beta-1}\right)=O\left(n^{1-\beta} \theta^{-(1-\beta) n}\right),
$$

and Theorem 11 is proved.

Corollary. If $f(z)=\sum_{n=1}^{\infty} a_{n} e^{-\lambda_{n} z}$ with $\lambda_{n+1} / \lambda_{n} \geqq \theta>1$ converges for $\operatorname{Re} z=x>0$ and is strongly continuous as $z=x \rightarrow 0+$ in the sense of (4.10), then $f$ is of bounded variation in $(0,1)$.

This follows from

$$
\begin{aligned}
\int_{0}^{1}\left|f^{\prime}(x)\right| d x & =\int_{0}^{1}\left|\sum_{n=1}^{\infty} a_{n} \lambda_{n} e^{-\lambda_{n} x}\right| d x \\
& \leqq \int_{0}^{1} \sum_{n=1}^{\infty}\left|a_{n}\right| \lambda_{n} e^{-\lambda_{n} x} d x=\sum_{n=1}^{\infty}\left|a_{n}\right| \lambda_{n} \int_{0}^{1} e^{-\lambda_{n} x} d x \leqq \sum_{n=1}^{\infty}\left|a_{n}\right|
\end{aligned}
$$

and Theorem 11.

Finally we remark that $f(t)=O(1 / t), t \rightarrow 0+$, implies

$$
a_{n}=O\left(\lambda_{n} \log n\right), \quad n \rightarrow \infty,
$$

which is slightly better than the result in $\S 4.2(\mathrm{~b})$; this is obtained by 
putting $T=\lambda_{n}{ }^{-1}$ in (4.7). If $f$ grows faster than $t^{-1}, t \rightarrow 0+$, our refined method does not improve the results obtained in $\$ 4.2$.

\section{REFERENCES}

[1] V. Bernstein, Leçons sur les progrès récents de la théorie des séries de Dirichlet, Paris, 1933.

[2] R. P. Boss, Entire Functions, Academic Press, New York, 1954.

[3] J. A. Clarkson and P. Erdös, Approximation by polynomials, Duke Math. J., 10 (1943), pp. 5-11.

[4] A. Edrei, Gap and density theorems for entire functions, Scripta Math., 23 (1958), pp. 117-141.

[5] D. GAIER, Der allgemeine Lückenumkehrsatz für das Borel-Verfahren, Math. Z., 88 (1965), pp. 410-417.

[6] G. H. Hardy, Divergent Series, Clarendon Press, Oxford, 1949.

[7] J. KorevaAr, A characterization of the sub-manifold of $C[a, b]$ spanned by the sequence $\left\{x^{n_{k}}\right\}$, Nederl. Akad. Wetensch. Proc. Ser. A, 50 (1947), pp. 750-758.

[8] B. Kutrner, A theorem on Abel summability, J. London Math. Soc., 37 (1962), pp. 123-125.

[9] A. J. Macintyre, Asymptotic paths of integral functions with gap power series, Proc. London Math. Soc. (3), 2 (1952), pp. 286-296.

[10] W. Meyer-König and K. Zeller, Lückenumkehrsätze und Lückenperfektheit, Math. Z., 66 (1956), pp. 203-224.

[11] G. Pólya, Untersuchungen über Lücken und Singularitäten von Potenzreihen, Ibid., 29 (1929), pp. 549-640.

[12] G. Pólya and G. Szegö, Aufgaben und Lehrsätze aus der Analysis, vol. II, Springer, Berlin, 1925.

[13] A. Zygmund, On certain integrals, Trans. Amer. Math. Soc., 55 (1944), pp. 170204. 